\title{
ASPECTOS DE ALEVINAJE DE LAS PRINCIPALES \\ ESPE CIES NATIVAS LTTIHZADAS EN PISCICULTURA \\ EN LA AMAZONIA PERUANA
}

\author{
ALCANTARA BOCANEGRA FERNANDO $\left({ }^{*}\right)$ \\ GUERRA FLORES HUMBERTO(*)
}

\section{RESUMEN}

Los alevinos de las principales especies nativas utilizadas en piscicultura en la amazonia peruana, son recolectados anual mente de sus hábitats naturales al inicio de la creciente de las aguas.

Durante los años 1977, 1978 y 1979, se efectuaron observaciones de campo en el área de estudio (Fig. 1) en relación a las áreas, métodos de captura y transporte de los alevinos de las especies gamtana, Colossoma macropomun; paco, Colossoma brachypomum sábalo cola roja, Brycon sp. y boquichico, Prochilodus nigrcans.

Las capturas se efectuaron en los remansos, playas, charcos, caño y ambientes típicos más adecuados de los meandros de los ríos A mazonas y $\mathrm{N}$ apo (Fig. 2).

Se observó tolerancia a temperaturas altas ( $39^{\circ} \mathrm{C}$ ), en las especies boquichico, gamitana, paco, sábalo y sardina (Tabla 2), así como a bajos niveles de oxígeno disuel to $(0.8 \mathrm{ppm})$, en gamitana, paco, sábalo y otras especies (Tabla 3).

Los alevinos de sábalo, comprendidos entre 15 y $65 \mathrm{~mm}$, tienen preferencia por los insectos, siguiendo en orden de importancia el zooplancton, las larvas de peces, el fitoplancton y las macrófitas. 
La vegetación herbácea, ribereña como la vegetación flotante, tienen gran importancia en la supervivencia de los alevinos, pues no sólo les proporcionan alimento directa o indirectamente, sino también refugio.

En la fase de alevinaje, las especies consideradas presentan características propias que las diferencian de los adultos; en tal razón, se propone una clave de campo para su identificación.

Palabras claves: Alevinos de gamitana, Colossoma macropomum; paco C. brachypomum; sábalo cola roja, Brycon sp.; boquichico, Prochilodus nigricans. Observaciones ecológicas.

\section{INTRODUCCION}

Desde la década del sesenta, se están .cultivando en la región de la amazonía peruana, algunos peces tropicales nativos con fines de alimentación. Entre las especies que han recibido mayor atención están: la gamitana, Colossoma macropomum; el paco, Piaractus brachypomum; el boquichico Prochilodus nigricans y el sábalo cola roja, Brycon sp.

Durante los primeros años, el aprovisionamiento de alevinos de estas especies se realizó exclusivamente del medio natural y, a la fecha, se realiza tanto de los ambientes naturales, como de la producción en condiciones controladas, según se trate de gamitana o paco (A LCANTARA, 1985; ALCANTARA y GUERRA, 1986).

Se conoce muy poco acerca de los alevinos de las especies de interés para la piscicultura y este conocimiento se encuentra a nivel de observaciones personales. Al mismo tiempo, dado el carácter aleatorio de la distribución espacial y temporal de las especies en el medio natural, el aprovisionamiento de una buena cantidad de alevinos se presenta como una limitante del desarrollo de la acuicultura regional, que deberá superarse a medida que se logre reproducir en condiciones controladas otras especies, además de gamitana y paco, así corno se alcancen adecuados niveles de sobrevivencia de alevinos.

Este trabajo tuvo como objetivos el estudio de las áreas naturales de alevinaje, así como el estudio morfológico de los alevinos, considerando que la mayoría de especies de interés, en su fase de alevinos, presentan algunas características diferentes a las de los adultos. 


\section{MATERIAL Y METODOS}

Durante el período comprendido entre los meses de octubre a febrero de los años 1977, 1978 y 1979, con ocasión de recolectar alevinos de las especies gamitana, Colossoma macropomum, paco, Colossoma brachypomum, sábalo cola roja, Brycon sp. y boquichico, Prochilodus nigricans, con fines de cultivo, se efectuaron observaciones en el medio natural referentes a las áreas y métodos de captura y transporte de estos alevinos.

El área geográfica de muestreo abarcó un tramo del río Amazonas, comprendido entre $\mathrm{M}$ uyuy y la desembocadura del río $\mathrm{Napo}$, aguas arriba y aguas abajo de Iquitos, respectivamente (Fig. 1); y el río $\mathrm{Napo}$, desde su desembocadura hasta A tún Cocha.

Las zonas de muestreo no se eligieron al azar, estuvieron en cierto modo condicionadas por nuestra necesidad de captura de alevinos de gamitana. No obstante, en cada ocasión se siguió un procedim\$ento uniforme, que consistió en registros de datos físico-químicos, determinación de la composición de la captura, en base a una cala, y determinación de la mortalidad por pesca y por transporte.

Se efectuaron análisis de oxígeno disuelto, anhidrido carbónico y pH, en adición de los registros de temperatura y trasparencia.

El oxígeno disuelto y el anhidrido carbónico se determinaron mediante el Laboratorio Portátil para Análisis Químicos de Agua: LAB - KIT 1, el pH se determinó mediante el Comparador Universal de $\mathrm{pH}$ Merck, los registros de temperatura se efectuaron mediante un termómetro simple, graduado al $0.1^{\circ} \mathrm{C}$ y la transparencia, mediante el disco Secchi.

Para la captura se utilizó una red pequeña de $2.5 \mathrm{~m}$ de largo por $1.20 \mathrm{~m}$ de al to, con malla de $1 \mathrm{~mm}$. La red fue operada por dos hombres, generalmente en aguas someras, con profundidades de 30 a $443 \mathrm{~cm}$. y siempre arrastrárfdola contra la orilla. En casos en los que la orilla estaba cubierta de vegetación herbácea o de tallos de arroz en putrefacción se operó la red con tres hombres, para evitar que la vegetación enrolle la red, tirando uno de ellos del centro de la relinga inferior de la red.

Para el almacenamiento y transporte se utilizaron cajas isotérmicas de tecnoport de $0.40 \times 0.40 \times 0.20 \mathrm{~m}$, y no se emplearon tranquilizantes. En algunos casos fue necesario cambiar el agua de las cajas al término de la operación de captura. 
Para el traslado de los peces se utilizó una embarcación pequeña y veloz (deslizador), equipada de un motor fuera de borda.

FIG. 1

AREA DE ESTUDIO

1 Padre Isla

2 Isla L upuna

3 M uyuy

4 Pihuicho Isla

5 Panguana

6 Iquitos

7 M oena C año
8 Petrolera

9 Timicurillo

10 Indiana

11 Timicuro

12 Isla M arupá

13 Orellana

Escala 1:500,000

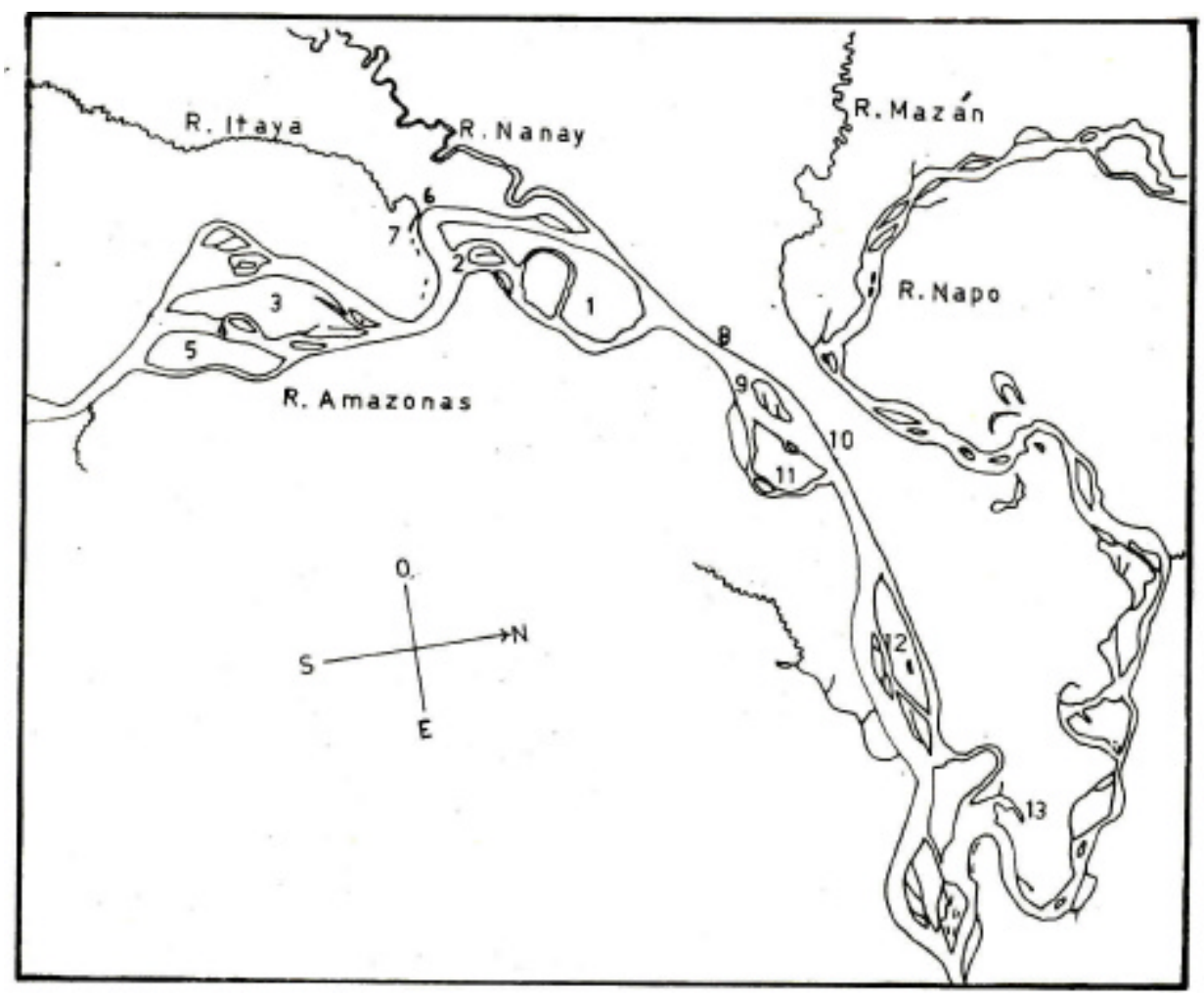




\section{RESULTADOS Y DISCUSION}

\section{A. Morfología de las áreas de alevinaje.}

Los ambientes explorados para la captura de alevinos estuvieron comprendidos en cuatro categorías: remanso, playa, charco y caño, ambientes que se repiten a través de los meandros que describe el canal principal del río en su recorrido (Figura 2).

Los remansos se caracterizan por ser brazos pequeños abiertos del río, de aguas quietas y someras.

L as playas son áreas convexas que se forman en la orilla interior del meandro y se caracterizan por presentar los suelos de arena o sedimentos finos, de suave declive.

L os charcos son cochas o pantanos pequeños, formados porinundación de las depresiones del terreno, durante las oscilaciones de la creciente de los ríos.

L os caños son canales pequeños laterales al canal principal.

Dos procesos geomórficos predominan en las orillas a lo largo del canal. En la orilla exterior, de naturaleza cóncava, predomina la erosión, dando lugar a la formación de barrancos o zonas de costa brava. En la orilla interior convexa, predomina la sedimentación y drenamiento. La sedimentación da lugar a la formación de playas $y$, en partes, a les ten nos denominados localmente como barriales, que se utilizan en el cultivo de arroz.

\section{B. Hidrología}

Los niveles del río A mazonas presentan variaciones frecuentes a través de todo el año; los niveles máximos se observan entre los meses de abril y mayo y corresponden al período de "creciente" 0 de expansión, en que las aguas inundan la llanura. Los niveles mínimos se observan entre los meses de setiembre y octubre y corresponden al período de "vaciante" o de retracción, en que las aguas vuelven a su cauce. (Figura 3).

En la zona de Iquitos, durante los meses de octubre-noviembre, se produce una pequeña creciente del río A mazonas y simultáneamente aparecen los alevinos de las principales especies de Carácidos. Esto vendría a ser la evidencia local de que... "casi todas las especies de peces de los ríos de tierras de inundación cambian de 
comportamiento con las estaciones, reproduciéndose al principio de las inundaciones" (W elcome, 1980).

FIG. 2

CARACTERISTICAS MORFOLOGICAS DE LAS AREAS DE ALEVINAJE

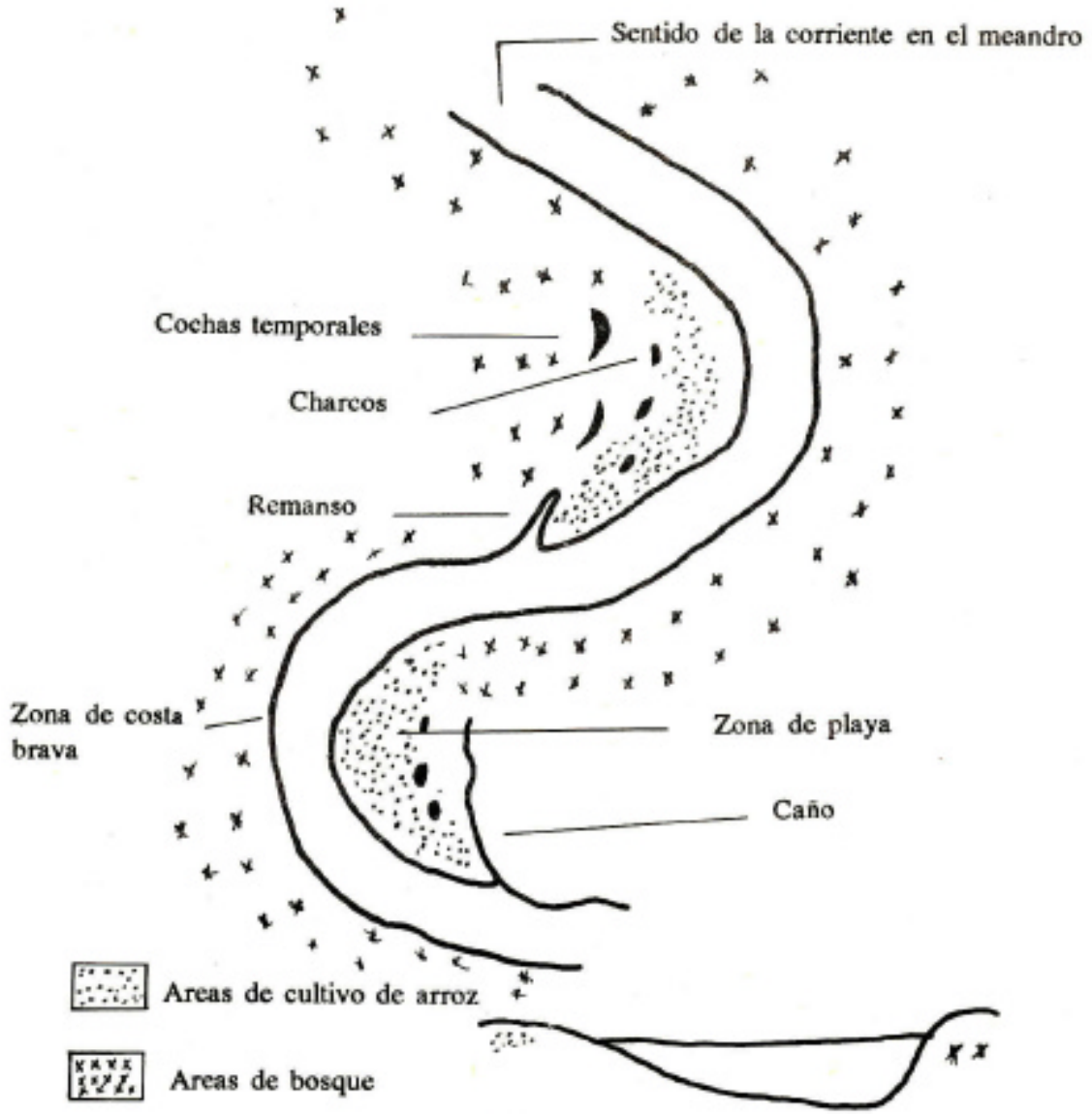


FIG. 3

NIVELES DEL AMAZONAS

(Guerra et al. 1981)

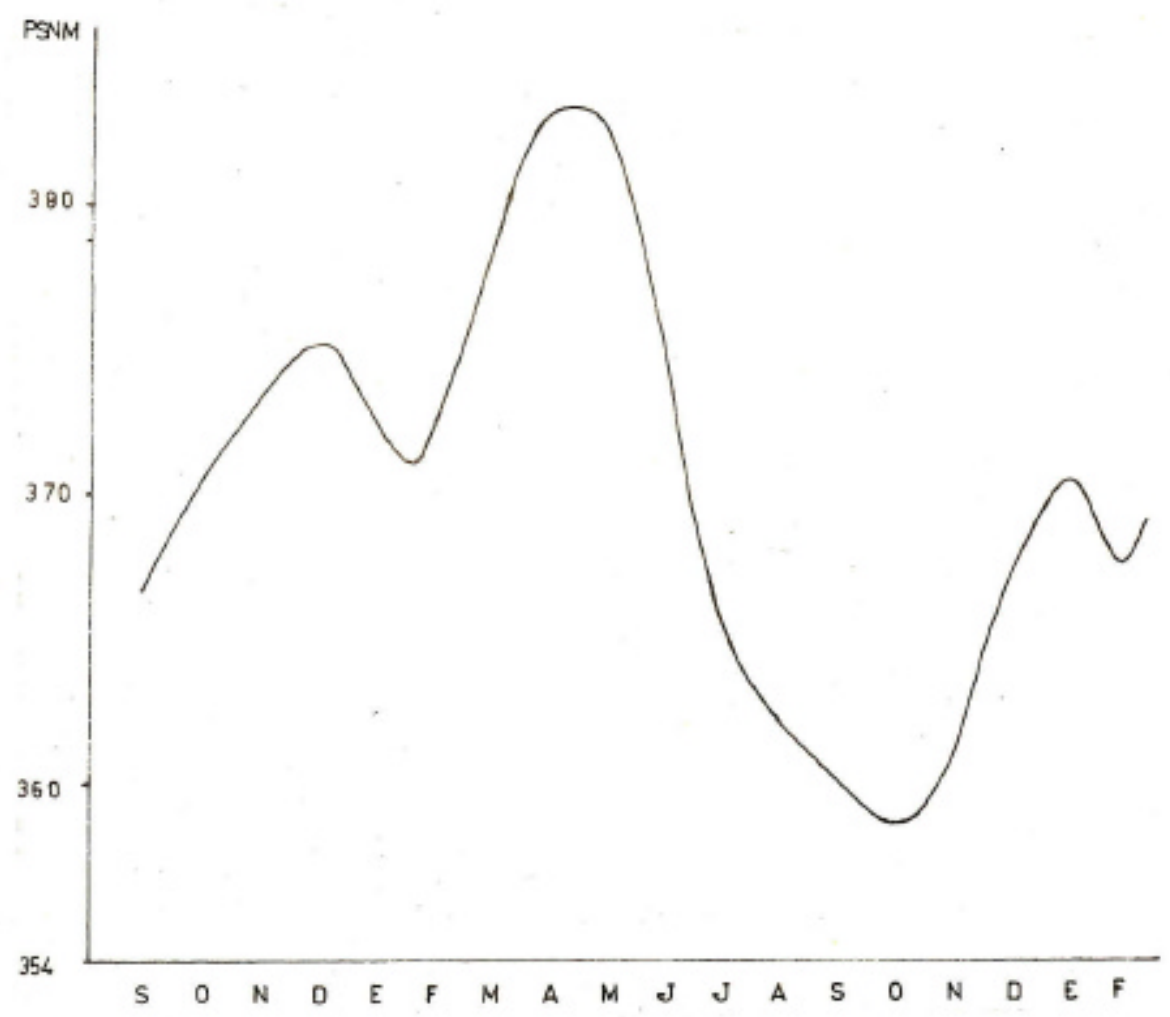

Al producirse el incremento de nivel del río se van inundando las depresiones de la Ilanura, las que quedan como cuerpos de agua pequeños y temporales, aislados del cauce del río, cuando se produce una disminución del nivel o cuando cambia de curso. La formación de estos cuerpos de agua temporales es frecuente, ya que, como es sabido, la creciente del río no se presenta como una constante, observándose por el contrario, dentro de ella, fluctuaciones frecuentes. Los ambientes formados por inundación alienen los alevinos, hasta que el río vuelve a incrementar su nivel; pero, esto lógicamente depende de sus dimensiones, así como del tiempo en que el río vuelve a alcanzar su nivel de inundación original, de otro modo, desaparecen. En varias ocasiones se efectuó la localización de cuerpos de agua en vías de desaparición, 
detectables por la presencia de gran número de aves piscívoras alimentándose de alevinos.

C. Factores físico-químicos

$\underline{\text { TABLA } 1}$

FACTORES FISICOS Y QUIMICOS EN LAS AREAS DE ALEVINAJE

\begin{tabular}{|l|l|l|l|ll|l|}
\hline & Temperatura & Transparencia & Tipo de agua & $\begin{array}{l}\mathrm{O}_{2} \\
\mathrm{Ppm}\end{array}$ & $\begin{array}{l}\mathrm{CO}_{2} \\
\mathrm{ppm}\end{array}$ & $\mathrm{pH}$ \\
\hline Rango & $26.0-39.0$ & $0.1-10$ & Blanca-negra & $0.8-9.6$ & $2-23$ & $6.0-7.5$ \\
\hline
\end{tabular}

Con excepción de la temperatura y del oxígeno, los factores físicos- químicos observados estuvieron comprendidos entre límites normales. La Tabla 1 muestra los valores e remos registrados.

$L$ a resistencia a las temperaturas elevadas es muy acentuada, tal es el caso de las especies que se capturaron en un pequeño charco con una temperatura de $39.0^{\circ} \mathrm{C}$, como se señala en a Tabla 2.

La resistencia a los bajos niveles de oxígeno disuelto es también elevada, tolerando niveles tan bajos como de $0.8 \mathrm{ppm}$, como en el caso de as especies que se capturaron en las playas 0 en los remansos (Tabla 3), que representaba abundantes cantidades de tallos de arroz en putrefacción. En estos ambientes, que antes del inicio de la creciente fueron campos de cultivo de arroz, es costumbre en la región, luego de efectuada la cosecha, dejar los tallos de arroz cortados y tendidos formando círculos de más o menos $0.80 \mathrm{~m}$ de diámetro y cuando el agua de la creciente inunda estos campos, se inicia una fuerte descomposición de la materia orgánica, favoreciendo una elevada proliferación de microorganismos (algas, ciliados, rotíferos, cladóceros) y de algunos insectos (Dípteros, hemípteros, coleópteros y odonatos), que en su mayoría son utilizados como alimento por los alevinos. 


\section{TABLA 2}

\section{ESPECIES QUE SE REGISTRARON \\ EN MEDIOS CON ALTA TEMPERATURA \\ (39 C). (M uestreo de una cala)}

\begin{tabular}{cccc}
\hline & L ongitud total & \\
Especie & $\mathrm{mm}$ & $\mathrm{N} \cong$ & $\%$ \\
\hline Prochilodus nigrigans & 35 & 12 & 23 \\
Colossoma macropomum & 30 & 10 & 20 \\
$\frac{\text { Colossoma brachypomum }}{\text { Brycon sp. }}$ & 30 & 6 & 12 \\
Triportheus sp. & 35 & 8 & 16 \\
\hline
\end{tabular}

D. Vegetación

La vegetación acuática propia estuvo representada por Pistia stratiotes Limnobium sp., Eichornia crassipes, E. azurea y Salvinia sp.

Entre la vegetación marginal más representativa se observó arroz en rebrote o tallos en putrefacción, luego de la cosecha; gramalote Paspalum sp., y Echinocloa sp., maiz y algunas gramíneas pequeñas. Es necesario señalar que solo se consideró, para el caso, la vegetación marginal directamente asociada al cuerpo de agua al inicio de la creciente; en tal sentido, la exclusión de la vegetación arbustiva y arbórea, es obvia. La razón de esta determinación está en que en el apogeo de la creciente, cuando la inundación alcanza el bosque, la captura de los al evinos es virtualmente nula.

\section{E. Alimentos y hábitos alimentarios.}

En la Tabla 4 se observa que los alevinos de sábalo, con una talla comprendida entre 1.5 y6.5 cm., tienen una alta preferencia por los insectos (61\%), observándose entre ellos: dípteros (Chironomidae), hemípteros, coleópteros, odonatos (ninfas), efemerópteros e himenópteros. 


\section{TABLA 3}

ESPECIES OBSERVADAS QUE TOLERAN

BAJ OS NIVELES DE 02 DISUELTO (0.8 ppm).

(M uestreo de una cala).

\begin{tabular}{|c|c|c|c|}
\hline \multicolumn{4}{|c|}{ L ongitud total } \\
\hline Especie & $\mathrm{mm}$ & N은 & $\%$ \\
\hline Crenicichla sp. & 45 & 64 & 30.0 \\
\hline Synbranchus marmoratus & 45 & 19 & 8.6 \\
\hline Colossoma macropomum & 45 & 18 & 8.4 \\
\hline$\overline{\text { Colossoma brachypomum }}$ & 40 & 46 & 21.5 \\
\hline Leporinus sp. & 50 & 2 & 1.0 \\
\hline Tetragonopterus sp. & 50 & 4 & 2.0 \\
\hline M ylossoma sp. & 40 & 1 & 0.5 \\
\hline Brycon sp. & 50 & 33 & 15.4 \\
\hline Gasterotornus sp. & 35 & 3 & 1.4 \\
\hline Semaprochilodus insignis & 50 & 24 & 11.2 \\
\hline
\end{tabular}

En orden de importancia, luego de los insectos, están el zooplancton, las larvas de peces y/o alevinos, el fitoplancton y las macrófitas. El porcentaje de estómagos observados conteniendo larvas de peces y/o alevinos mostró que esta especie tiene tendencia hacia la predación y al canibalismo, confirmándose esto a través de observaciones en condiciones controladas. Entre las macrófitas observadas en el contenido estomacal están: granos de arroz y semillas de gramíneas.

En relación a los Colossoma, las observaciones del contenido estomacal efectuadas en especímenes de 1.5 a $4 \mathrm{~cm}$., mostraron también preferencia por los insectos y zooplancton. 


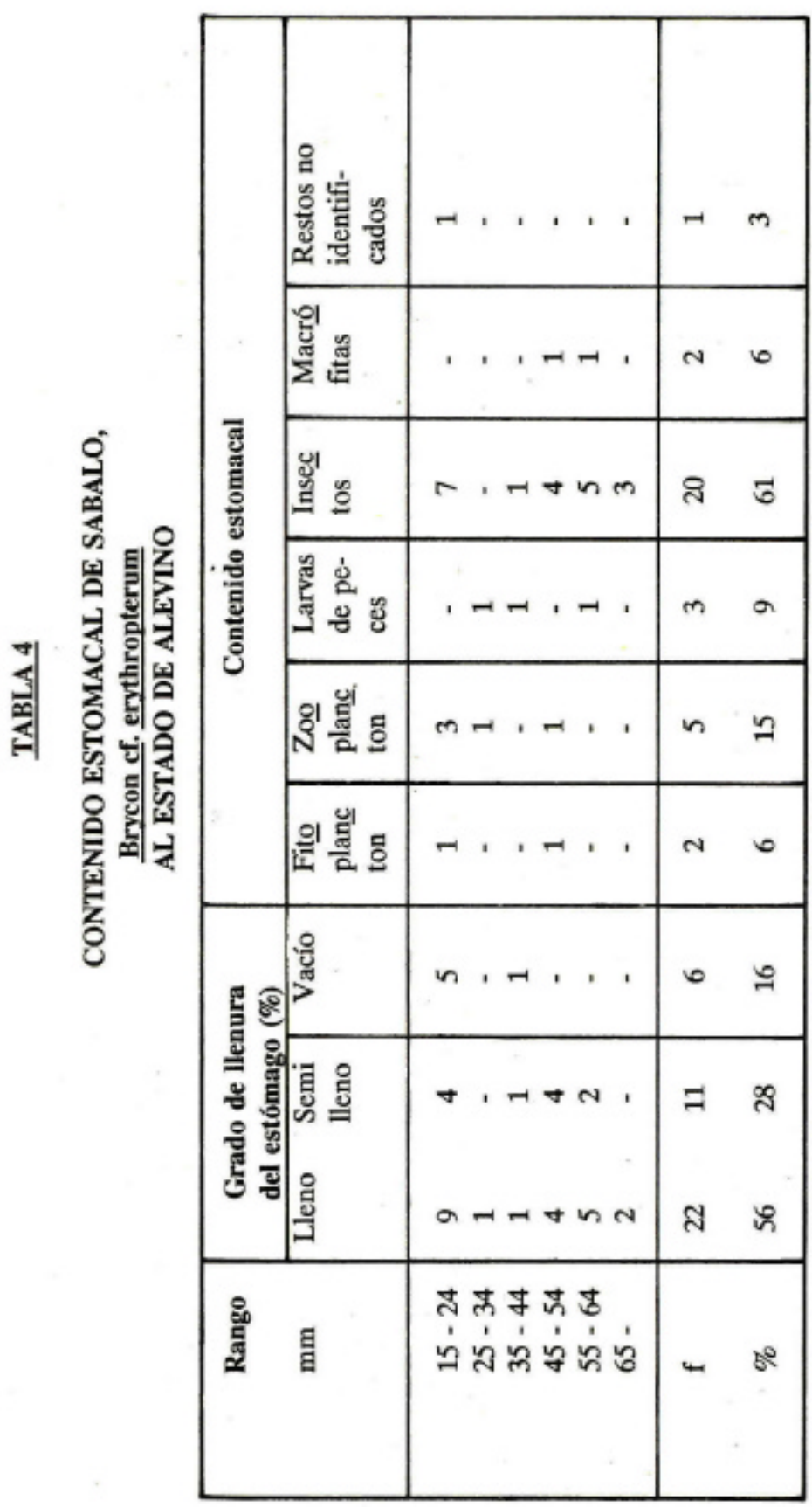




\section{F. Observaciones ecológicas}

Las especies nativas que se estén utilizando en piscicultura en la amazonía peruana, son especies reofílicas, que se reproducen al inicio de la creciente en los cursos principales de los ríos.

Poco antes de la estación lluviosa, gran número de carácidos migran contra la corriente, entre ellos importantes especies, tales como Prochilodus, M yleus, M etynnis y Triportheus. Estos peces desovan siguiendo el inicio de las Iluvias y el incremento en el nivel del agua. Durante las migraciones de desove recorren grandes distancias, desde los cursos bajos y medios de los ríos hacia los cursos superiores, alcanzando aguas con temperaturas 4 으 menores que las de origen. La disminución de la temperatura del agua, causada por la lluvia, tiene un efecto estimulante definido en los peces amazónicos. Las lluvias, particularmente en las aguas negras, pueden tener un efecto indirecto en los peces, por el incremento en el contenido de oxígeno (Geisler, et al. 1973).

La gamitana, Colossoma macropomum, migra por las partes profundas del canal, generalmente no revela su presencia cuando pasa por las desembocaduras de los ríos; cuando no migra, permanece en las palizadas de las márgenes de los ríos (Goulding, 1979)

Los sábalos, Brycon sp, por el contrarío, migran en el estrato superficial del canal, describiendo círculos que Goulding (1979) denomina como "rodada".

Se conoce muy poco acerca de la biología de reproducción de Prochilodus, Colossoma macropomum C. Brachypomum y Brycon. Los Prochilodus forman cardúmenes compactos de hembras y machos, denominados localmente como 'mijano", pirasema' en Brasil y 'subienda" en Colombia; migran por las orillas contra la corriente, en busca de las áreas de reproducción. En su recorrido, los machos emiten ronquidos prolongados que son perfectamente audibles desde tierra. Este comportamiento de "mijano" es utilizado por los pescadores locales para lograr buenas capturas en poco tiempo, dándose el caso de que la población adulta de todo un caserío ribereño se traslade hacia los caños para esperar el "mijano" que sale de las lagunas.

A través de los experimentos de reproducción inducida llevados a cabo con estas especies por numerosos investigadores de la región (Ihering, 1932; 
Fontenele, 1935 y otros, citados en Alcántara 1985), se conoce que Prochilodus sp., Brycon sp., Colossoma macropomum y C. brachypomum, son peces de desove total. Los óvulos de la 'gamitana C. macropomum, según Woynarovich (1977), antes de hidratarse tienen un diámetro de $1.34 \mathrm{~mm}$, y luego de hincharse en agua alcanzan un diámetro de 2.7 a $2.8 \mathrm{~mm}$; los huevos son de tipo flotante y no adhesivos.

Las especies de Brycon, así como de muchos carácidos amazónicos son pelagófilas, de acuerdo a la clasificación de Balon (1975), citado por Eckmann (1984), y presentan dos adaptaciones importantes al estadio embrionario. Primero, el espacio perivitelino de los huevos se agranda rápidamente en contacto con el agua, reduciendo el peso específico e incrementando la resistencia al flujo, con lo cual se facilita la libre flotación de los huevos; segundo, el desarrollo embrionario es sumamente rápido.

En Prochilodus, Colossoma macropomum y $\mathrm{C}$. brachypomum, en condiciones de laboratorio, se ha observado que el proceso de hidratación es muy rápido y los huevos prácticamente duplican su diámetro en menos de cinco minutos. A simismo, el desarrollo embriológico es un proceso rápido y ocurre entre 15 a 20 horas, variando de acuerdo a la temperatura media de incubación. (Da Silva, 1977; Woynarovich, 1977; Pezo y Sicchar, 1979; Eckmann, 1980; Alcántara, 1982; Alcántara, 1985; A lcántara y Guerra, 1986).

Al producirse la eclosión, las larvas ganan las orillas, en donde se desarrolla tanto la vegetación herbácea ribereña como la vegetación acuática flotante de los remansos, playas, charcos y caños; ésta vegetación tiene gran importancia en la supervivencia de los alevinos, pues no solo les proporcionan alimento directa 0 indirectamente, si no también refugio; por ello, es muy común pasar la red por debajo de la vegetación flotante (Eichornia, Pistia, Limnobium), para capturar gamitana y paco.

$L$ a resistencia a las altas temperaturas y bajos tenores de oxígeno disuelto, tiene una gran importancia ecológica para las especies consideradas, pues les permite ocupar ambientes con intensa descomposición orgánica, ricos en plancton, con el cual inician su alimentación. Adicionalmente, todas las especies consideradas mostraron alta resistencia al manipuleo, hecho que tiene gran interés para la piscicultura. 


\section{G. Pesca y transporte}

La captura de los alevinos de las principales especies para la acuicultura se efectuó en toda el área considerada en los muestreos, con excepción del caserío Portugal y A tún Cocha, en el río Napo (Figura 1). En la Tabla 5, se señalan las capturas observadas en las 13 diferentes áreas, siendo necesario precisar que la composición de las capturas debe considerarse sólo como referenciales, ya que corresponden a diferentes períodos de alevinaje, así como a diferentes ambientes.

\section{H. Mortalidad por pesca y por transporte}

Tal como se muestra en las Tablas $6,7,8,9,10$ y 11 , se observa que tanto la mortalidad por pesca como por transporte de los alevinos de las especies consideradas tuvo baja incidencia; sin embargo, las pruebas efectuadas no permiten sugerir una cantidad de alevinos por caja, debido a que el número de peces está influenciado también por los factores ambientales, tales como la temperatura del agua, el tenor de oxígeno disuelto y el número de calas con que son capturados los peces, ya que el stress juega un papel importante en la sobrevivencia de los mismos.

\section{Clave de campo para los alevinos de las principales especies de cultivo de} la Familia Characidae.

a. Cuerpo comprimido, aleta anal bien desarrollada.

b. A bertura de la boca en vista lateral, en forma de ángulo agudo.

c. Lados del cuerpo con una mancha obscura bien conspícua, más o menos circular, con halo blanquesino, a la altura de la aleta dorsal (válido hasta los $10 \mathrm{~cm}$. de longitud).

cc. Lados del cuerpo plateado o con numerosas manchas oscuras, mandíbula inferior saliente, al ser extraídos del agua abren y cierran la boca vigorosamente, pedúnculo caudal con una banda negruzca, borde posterior de la aleta anal más o menos oblicuo al eje del cuerpo. Fig. 6. - Serrasalmus.

d. Borde posterior de la aleta anal, recto, más o menos perpendicular al eje del cuerpo, aleta anal color rojo sangre con bordes oscuros. Fig. 4.- Colossoma macropomum. 


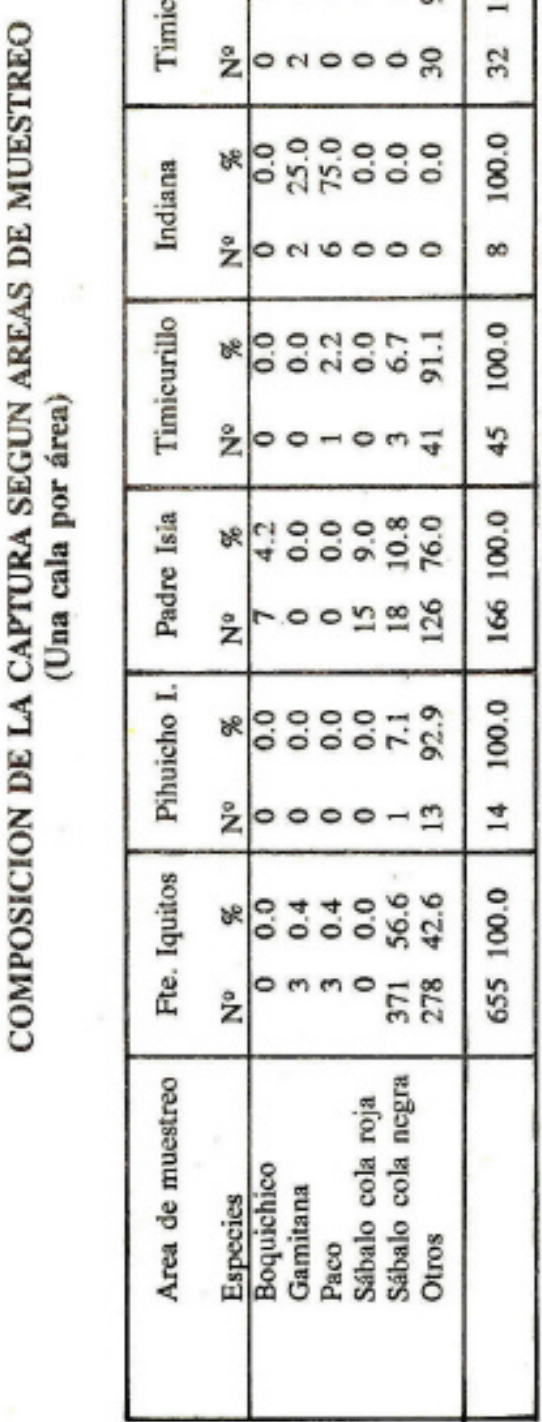

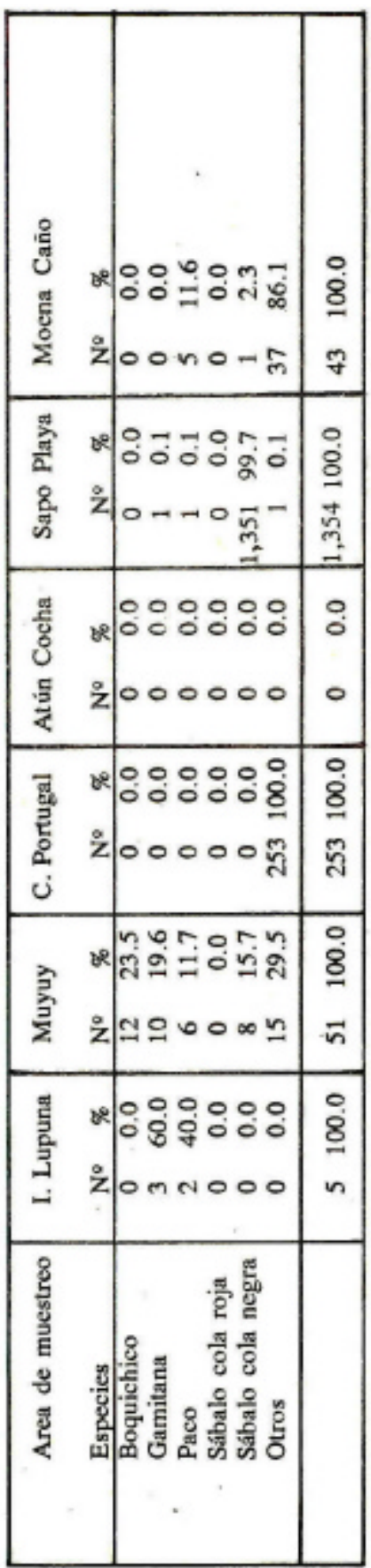



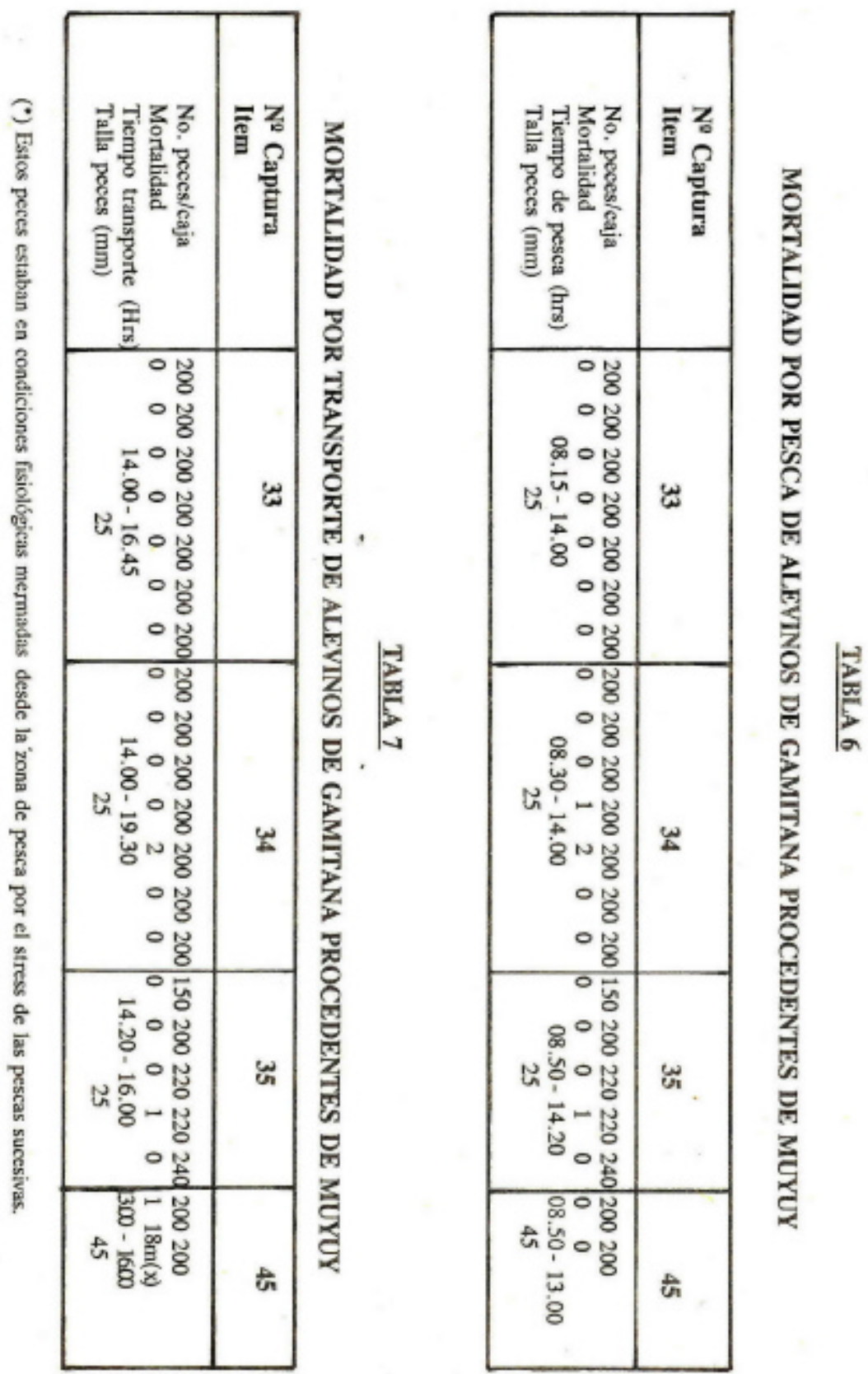
TABLA 8

MORTALIDAD POR PESCA DE ALEVINOS DE PACO PROCEDENTES DE MUYUY.

\begin{tabular}{ccccccc}
\hline № peces/caja & 200 & 200 & 220 & 240 & 260 & 280 \\
\hline M ortalidad & 0 & 0 & 0 & 0 & 1 & 0 \\
\hline Tiempo pesca (Hras) & \multicolumn{7}{c}{$08.50-13.00$} \\
\hline Talla peces $(\mathrm{mm})$ & & 40 \\
\hline
\end{tabular}

\section{$\underline{\text { TABLA } 9}$}

MORTALIDAD POR TRANSPORTE DE ALEVINOS DE PACO PROCEDENTES DE MUYUY.

\begin{tabular}{ccccccc}
\hline N - peces/caja & 200 & 200 & 220 & 240 & 260 & 280 \\
\hline M ortalidad & 0 & 1 & 0 & 1 & 1 & 0 \\
Tiempo pesca (Hras) & & & $13.00-16.00$ \\
Talla peces (mm) & & & & \\
\hline
\end{tabular}

\section{TABLA 10}

MORTALIDAD POR PESCA DE ALEVINOS DE SABALO DE COLA ROJA PROCEDENTES DE PIHUICHO ISLA

\begin{tabular}{ccccccc}
\hline № peces/caja & 281 & 420 & 450 & 475 & 540 & 595 \\
\hline M ortalidad & 1 & 10 & 22 & 55 & 8 & 28 \\
Tiempo pesca (H ras) & & & $11.00-12.30$ & & & \\
Talla peces (mm) & & & & \\
\hline
\end{tabular}


TABLA 11

\section{MORTALIDAD POR TRANSPORTE DE ALEVINO DE SABLO COLA ROJA PROCEDENTES DE PIHUICHO ISLA}

\begin{tabular}{ccccccc}
\hline $\mathrm{N}$ o peces/caja & 280 & 410 & 420 & 428 & 532 & 567 \\
\hline M ortalidad & 1 & 20 & 14 & 3 & 4 & 3 \\
Tiempo pesca (Hras) & & \multicolumn{7}{c}{$\begin{array}{c}13.05-16.50 \\
\text { Talla peces }(\mathrm{mm})\end{array}$} & & 20 & & \\
\hline
\end{tabular}

dd. Borde posterior de la aleta anal redondeado, aleta anal color anaranjado pálido. Fig. 5. - M ylossoma duriventris.

bb. A bertura de la boca en vista laterai en forma de ángulo agudo truncado.

e. $\quad$ Lados del cuerpo plateados sin manchas oscuras (mojarra). Fig. 9Tetraconopterus sp.

ee. $\quad$ Lados del cuerpo con numerosas manchas oscuras (válido hasta los $10 \mathrm{~cm}$. de longitud), borde posterior de la aleta anal mas o menos perpendicular al eje del cuerpo, sin banda negra en el pedúnculo caudal. Fig. 7. - (paco) Colossoma brachypomum.

aa. Cuerpo fusiforme, boca grande, no protráctil.

f. Boca extensible para formar un disco de succión; con una mancha negruzca de márgenes amarillentos en la aleta dorsal, cuando tienen longitudes de 15 a $20 \mathrm{~mm}$; con bandas claras y oscuras que se alternan a lo largo de todo el cuerpo, a excepción de la cabeza. Fig. 10.- (boquichico) Prochilodus.

ff. Boca no extensible, mancha negruzca mas o menos circular por detrás de la cabeza, sobre la

línea lateral,

g. Lóbulos de la aleta caudal blanquecinos o amarillentos, Fig $13 .$. (sábalo cola negra).

gg. Lóbulos de la aleta caudal rosados o rojizos.

Fih. A. (sábalo cola roja) Brycon erytropterum 

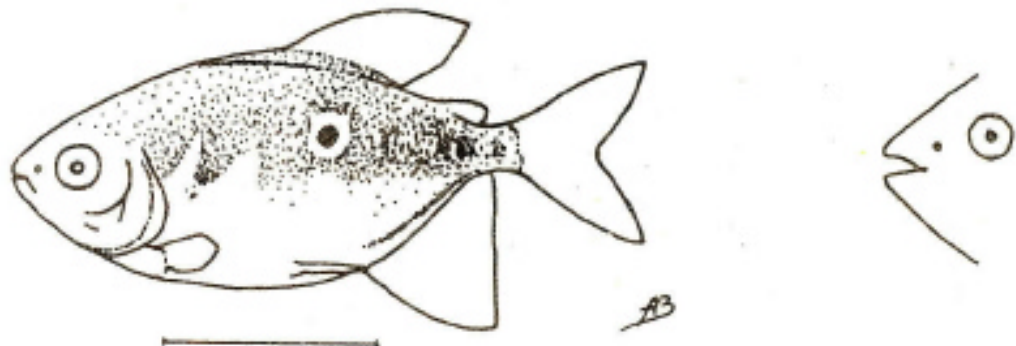

$10 \mathrm{~mm}$

Fig. 4 gamitana, Colossoma macropomum
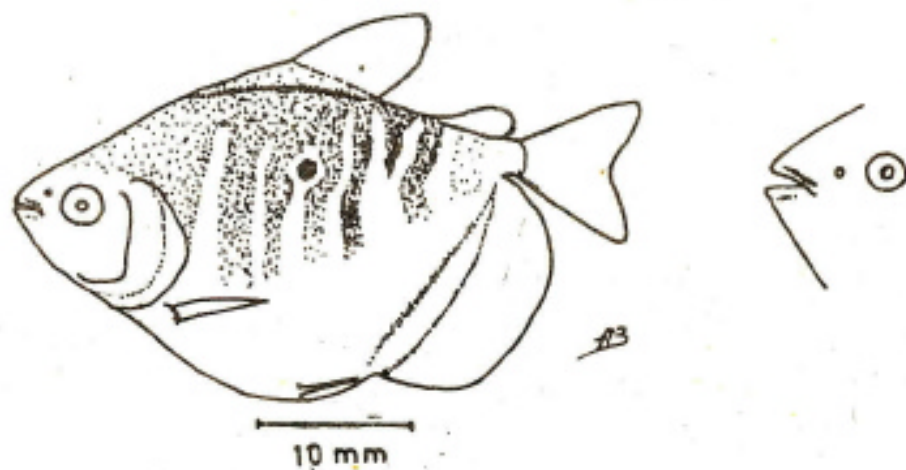

Fig. 5 palometa, Mylossoma duriventris
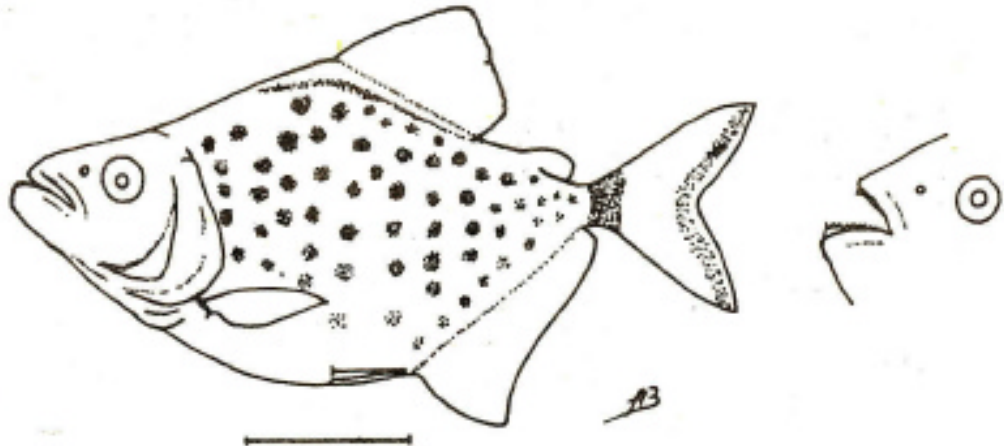

$10 \mathrm{~m} \mathrm{~m}$

Fig. 6 paña roja, Serrasalmus $\underline{\text { sp. }}$. 


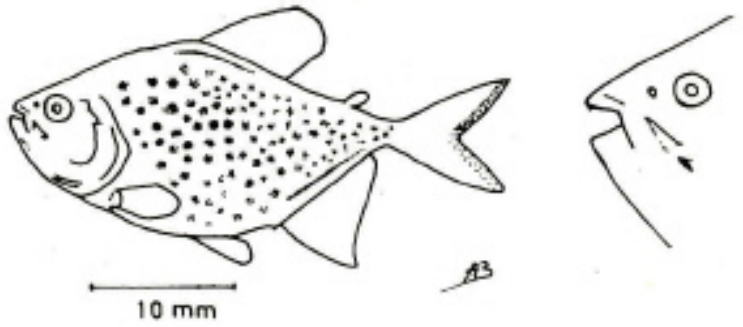

Fig. 7 paco, Colossoma brachypomum
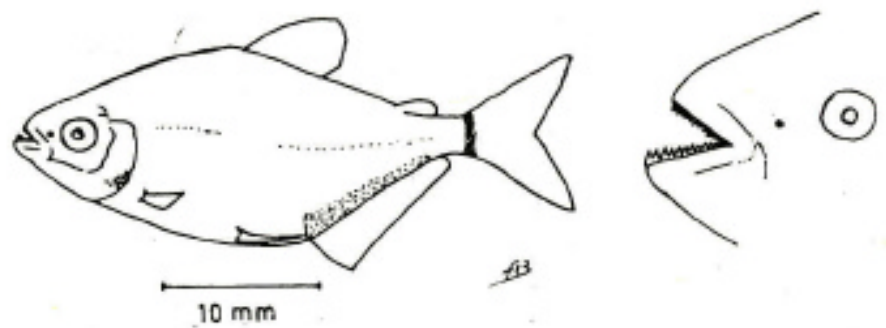

Fig. 8 paña blanca, Serrasalmus $\underline{\text { sp. }}$.
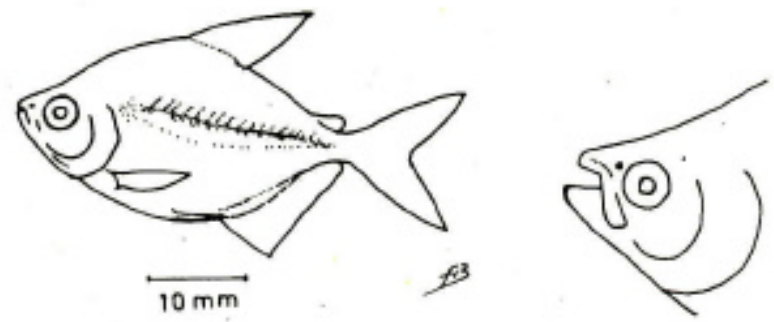

Fig. 9 mojarra, Tetragonopterus sp.

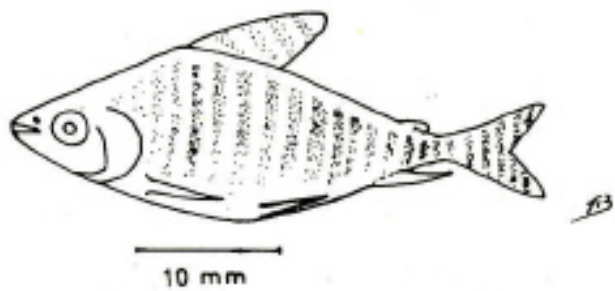

Fig. 10 boquichico, Prochilodus nigricans 


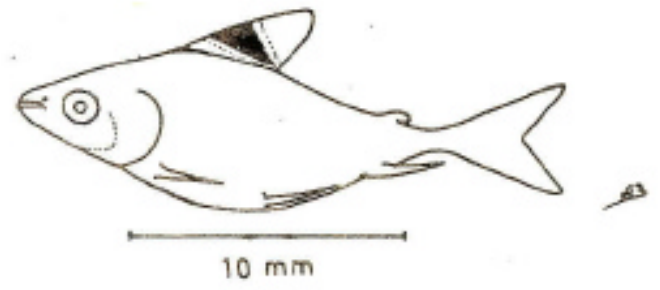

Fig. 11 boquichico, Prochilodus nigricans

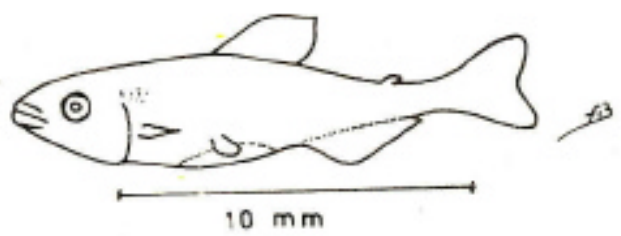

Fig. 12 sábalo, Brycơn $\underline{\text { sp. }}$.

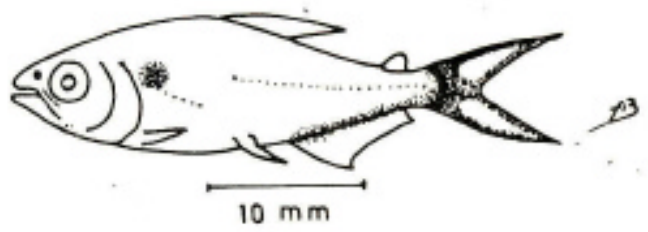

Fig. 13 sábalo cola negra Brycon melanopterum

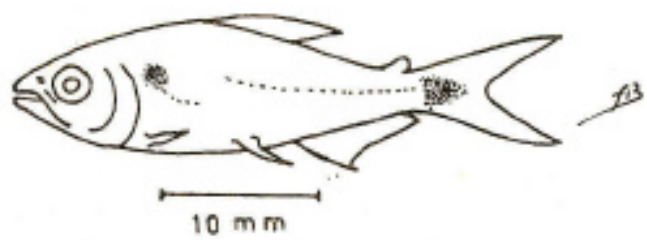

Fig. 14 sábalo cola roja $\underline{B}$. erythropterum 


\section{BIBLIOGRAFIA}

ALCANTARA, B.F., 1982. Ensayo sobre reproducción inducida de boquichico, Prochilodus nigricans, A gassiz 1829, en cautiverio. Informe interno. Inst. M ar del Perú - Laboratorio de Iquitos. 15 pp.

1985. Reproducción inducida de gamitana, Colossoma macropomum, Cuvier 1818 en el Perú. Tesis Doctoral. U niversidad Nacional de Trujillo. Trujillo Perú. 38 pp.

ALCANTARA, B.F. y H. Guerra F. 1986. A vances en la producción de alevinos de gamitana, Colossoma macropomum y paco $\mathrm{C}$. brachypomum por reproducción inducida. Rey. Lat. A eui. № 30, Lima. Perú.

BERMUDEZ, D. 1979. Observaciones sobre el desarrollo embrionario de la "cachama", Colossoma macropomum (Cuvier 1818). Universidad Centro Occidental. Escuela de Agronomía. Estación de Piscicultura. Barquisimeto. Venezuela. p. 16.

ECKMANN, R. 1980. Reproducción inducida de Prochilodus nigricans (Agassiz, 1829) del bajo A mazonas. Acuicultura. 20: 381-383. Elsevier Science Publishers. A msterdam.

1984. Induced Reproduction in Brycon ef. erythropterus. A quaculture. 38: 379-382. Elsevier Science Publishers. A msterdam.

EIGENMANN, C.H. 1912. The Fresh Water Fishes of British Guiana, including a study of the ecological gronping of species and the relation of the fauna of the plateau to that of the lowlands. M em. Carnegie M us. 5: $x x+578$ pp, 103 pls.

FONTENELE, 0. 1959. Injecting Pituitary (Hypophyseal). Hormones into Fish to Induce Spawning. Dept. Na. Obras contra as Secas. Brasil. Servicio de Piscicultura. Publicacao N 168. Serie 1-C pp 1 - 12.

GEISLER, R.; HA. K nóppel and H. Sioli. 1973. The ecology of Fresh Water Fishes in A mazonia. Present Status and Futurc Tasks for Rescarch. In Applied for Scientific Cooperation. pp. 144 - 62.

GOULDING, M. 1979. Ecología da pesca do Río Madeira. Trad. de Naercio M enezes. M anaus. JNPA. pp. 95 - 96, 154v - 156. 
GUERRA, F.H.; Víctor Montreuil F.; Marie Villacorta C. 1981. A vances del Programa de Evaluación de Recursos Pesqueros en la A mazonía Peruana. En Informe del Seminario de COPESCAL sobre Evaluación de las Pesquerías Fluviales y Grupo de Trabajo sobre Recursos Pesquerios, FAO. Colombia.

LOVSHIN, L.L.; Da Silva, A.B.; A. Carneiro Sobrinho; F.R. M elo. 1981. Biology and Culture Potential of Colossoma sp. Native to South A merica (M imeo). 16 pp.

PEZO, R.; L. Sicchar. 1979. Reproducción inducida por hipofisación en "boquichico Prochilodus nigricans, A gassiz 1829. Tesis para obtener el título de Biólogo. Universidad N acional de la A mazonía Peruana.

SIOLI, H. 1964. General features of the limnology of A mazonia. Verh. mt. Theor. A ugen. Lirnnol. 15, 1053 - p.

WELCOME. R.L. 1980. Cuencas fluviales. FA O, Doc. Tec. Pesca, (202) pp. 7 -9.

WOYNAROVICH, E. L'77. La propagaión de los peces. Dirección-General de Desarrollo Pesquero. Min. de Agricultura y Cría. Caracas. Venezuela. 7 pp. (M imeo). 